EPJ Web of Conferences 41, 04014 (2013)

DOI: $10.1051 /$ epjconf/20134104014

(C) Owned by the authors, published by EDP Sciences, 2013

\title{
Slow propagation of photon-like polaritons generated by exciton-exciton scattering in $\mathrm{ZnO}$ thin films
}

\author{
H. Ichida ${ }^{1}$, S. Wakaiki ${ }^{2}$, T. Kawase ${ }^{3}$, K. Mizoguchi ${ }^{4}$, D. Kim ${ }^{3}$, M. Nakayama ${ }^{3}$, and Y. Kanematsu ${ }^{1,2}$ \\ ${ }^{1}$ Venture Business Laboratory, Osaka University, 2-1 Yamada-oka, Suita, Osaka 565-0871, Japan \\ 2 Department of Material Life Science, Graduate School of Engineering, Osaka University, 2-1 \\ Yamada-oka, Suita, Osaka 565-0871, Japan \\ ${ }^{3}$ Department of Applied Physics, Graduate School of Engineering, Osaka City University, 3-3-138 \\ Sugimoto-cho, Sumiyoshi-ku, Osaka 558-8585, Japan \\ ${ }^{4}$ Department of Physical Science, Graduate School of Science, Osaka Prefecture University, 1-1 \\ Gakuen, Naka-ku, Sakai, Osaka 599-8531, Japan
}

\begin{abstract}
We report on the first observation of the thickness-dependent photoluminescence-decay time of exciton-exciton scattering in $\mathrm{ZnO}$ thin films, which indicates the slower propagation of photon-like polaritons compared to that in bulk by two orders magnitude.
\end{abstract}

The intense emission caused by exciton-exciton scattering, which is called $\mathrm{P}$ emission, is an interesting subject from an aspect of ultrafast phenomena leading to stimulated emission. Another attractive aspect of the $\mathrm{P}$ emission is that it enables us to access the related photon-like polariton state that can lead to slowdown of light propagation [1]. In the inelastic scattering process of two excitons in the first quantum $(n=1)$ state, one exciton is scattered into a higher excited state with $n \geq$ 2 , while the other exciton is scattered into a photon-like polariton state whose energy is lower than that of the $n=1$ exciton state by the energy difference between the $n=1$ and $n \geq 2$ states [2]:

$$
\operatorname{Ex}\left(n=1, k_{1}\right)+\operatorname{Ex}\left(n=1, k_{2}\right) \rightarrow \operatorname{Ex}\left(n \geq 2, k_{1}+k_{2}-k_{\mathrm{ph}}\right)+\operatorname{photon-like} \operatorname{polariton}\left(k_{\mathrm{ph}}\right),
$$

where $\operatorname{Ex}(n, k)$ represents the exciton in $n=\mathrm{i}$ exciton state with a wavenumber of $k$. In the dynamical property of the exciton-exciton scattering, we have to take into account at least two processes on the basis of the exciton-polariton picture. First, in the exciton-exciton scattering process, the two exciton-polaritons are converted into a higher exciton and a photon-like polariton when the exciton density is higher than the threshold density. The rate of this collision process depends on the exciton density. The second process is the transformation of the polaritons into external photons on the crystal surface. The rate of this process is determined by [3]

$$
\tau_{d}^{-1}=A \times v_{g}(E) \propto(1-R) / L \times v_{\mathrm{g}}(E) .
$$

Here, vg is the group velocity of the polaritons and $R$ and $L$ are the reflection coefficient and the film thickness, respectively. From Eq. (2), the lifetime of photon-like polariton is proportional to the group-velocity dispersion of the photon-like polariton branch. In our previous work, we reported that the decay time of the $\mathrm{P}$ emission changes with the detection energy and the energy dependence of that could be reproduced directly from the group-velocity dispersion of the photon-like polariton

This is an Open Access article distributed under the terms of the Creative Commons Attribution License 2.0, which permits unrestricted use, distribution, and reproduction in any medium, provided the original work is properly cited. 
branch [4]. However, the value of fitting parameter $A$ obtained from the data fitting of experimental results is much smaller than that calculated from the values of $R$ and $L$ of the $\mathrm{ZnO}$ thin film. The detail of the relationship between the decay time of the P-emission and the lifetime of the photonlike polariton remain to be elucidated.

In this study, to understand the detail of the lifetime of the photon-like polariton state, we have investigated the ultrafast dynamics probed via the time-resolved PL spectra of the P emission process in $\mathrm{ZnO}$ thin films with the film thickness of 200 and $500 \mathrm{~nm}$ by using an optical-Kerr gating method at $10 \mathrm{~K}$ from the viewpoint of the propagation of the photon-like polaritons.

The samples used is a crystalline $\mathrm{ZnO}$ thin films grown on a $(0001) \mathrm{Al}_{2} \mathrm{O}_{3}$ substrate at $650{ }^{\circ} \mathrm{C}$ prepared by a pulsed laser deposition (PLD) method. Time-resolved PL spectra were measured by using the following optical-Kerr-gating method. The excitation energy and the excitation density were $3.59 \mathrm{eV}$ and $65 \mu \mathrm{J} / \mathrm{cm}^{2}$, respectively. The detail of the experimental set up for OKG method is reported in Ref. 4.

Figure 1(a) and (b) shows the image plot of the time-resolved PL spectra in $\mathrm{ZnO}$ thin films with the film thickness of 200 and $500 \mathrm{~nm}$, respectively. In the time-integrated PL spectra depicted by the solid curve, two PL bands labelled P and M were observed. From the emission energy, we decided that the $\mathrm{P}$ - and $\mathrm{M}$-emission bands are originated from the exciton-exciton scattering and the biexciton in $\mathrm{ZnO}$, respectively. Figure 2(a) and (b) show the decay profile of the P- and M-emission bands at the various detection energies in $\mathrm{ZnO}$ thin films with the film thickness of 200 and $500 \mathrm{~nm}$, respectively. First, we focus on the decay profile at the detection energy of $3.345 \mathrm{eV}$ whose energy is agree with the M-emission-peak energy, which shows in the bottom of Figure 2 (a) and (b). The decay time of $\mathrm{M}$ emission is estimated about 25 ps from the data fitting shown by the dashed line. This result indicates that the decay time of the biexciton is independent of the film thickness of 200 and $500 \mathrm{~nm}$. On the other hand, the decay profile in the energy region observed the $\mathrm{P}$ emission change with the detection energy and the film thickness. In the detection energy from 3.305 to 3.265 $\mathrm{eV}$, we found that the decay profile is composed of the fast and slow components in Figure 2(a) and (b). In this energy region, the $\mathrm{P}$ emission is main component of the time-resolved PL spectra. Therefore, the fast component is considered to be due to the $\mathrm{P}$ emission. Under the week excitation conditions, the low energy tail of the $\mathrm{M}$ emission is observed in the energy region located the $\mathrm{P}$ emission, which is not shown here. From this fact, the slow component is considered to be due to the M-emission. The decay time of the slow component becomes faster with a decrease of the detection energy. We consider that this result reflects the temporal change of the M-emission band, which will be reported elsewhere. Here, we focus on the decay time of the fast component originated from the $\mathrm{P}$ emission. The decay time of that becomes faster with a decrease of the detection energy. In addition, it is found that the decay time of the P-emission band of $200 \mathrm{~nm}$ thin film is shorter than that in 500 $\mathrm{nm}$ thin film at the same detection energy.

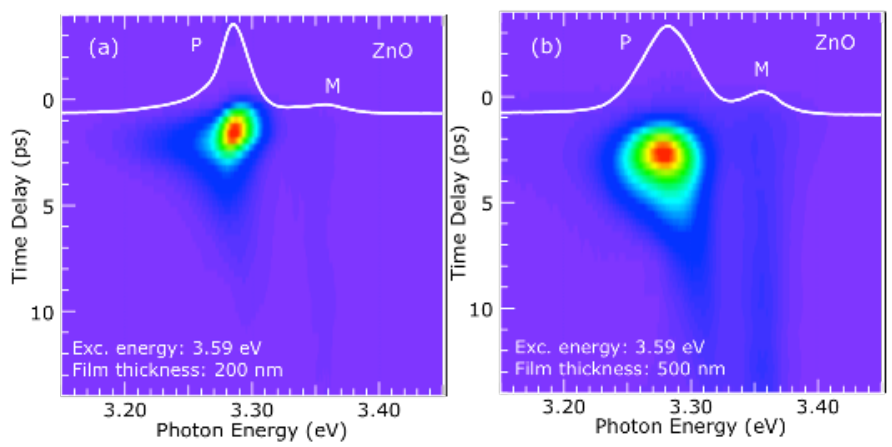

Fig. 1. Image plot of the time-resolved PL spectra in $\mathrm{ZnO}$ (a) $200 \mathrm{~nm}$ and (b) $500 \mathrm{~nm}$ thin films under the excitation power of $65 \mu \mathrm{J} / \mathrm{cm}^{2}$. The solid curve indicates the time-integrated PL spectra. 

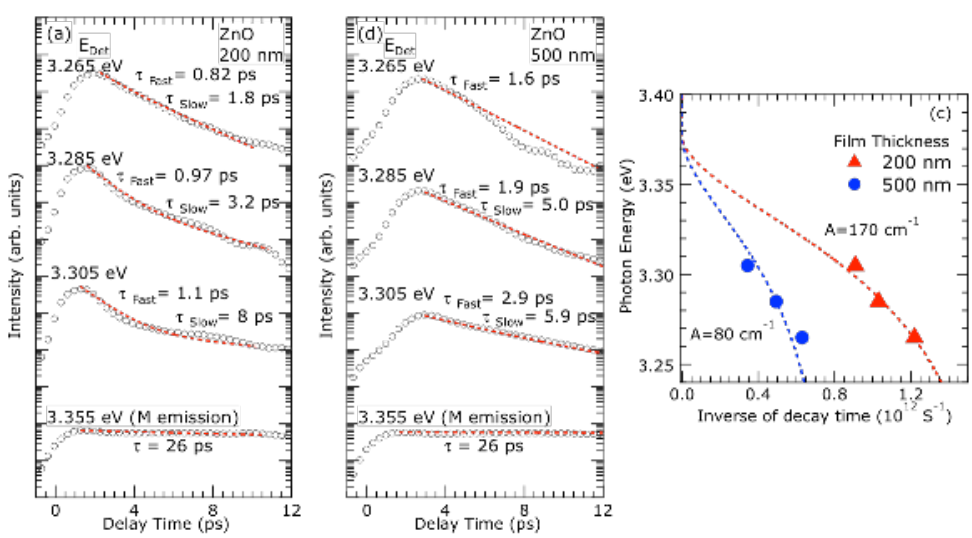

Fig. 2. The temporal profile of the PL intensity at various detection energies in (a) $200 \mathrm{~nm}$ and (b) $500 \mathrm{~nm}$ thin films. The dashed lines show the fitted results to the temporal profile in the PL decay region. (c) Relation between the inverse of the decay time of the $\mathrm{P}$ emission and the group velocity of the photon-like polariton. The dashed curve depicts the fitted result of the energy dependence of the group velocity on the basis of Eq. (2).

In order to elucidate the above properties of the decay profiles of $\mathrm{P}$ emission, we discuss the relation between the decay time of the $\mathrm{P}$ emission and the group velocity of the photon-like polariton based on Eq. (2). In Figure 2 (c), the circles and triangles indicate the inverse of the decay time of the P emission in 200 and $500 \mathrm{~nm}$ thin films, respectively, while the dashed curves show the fitted results of the energy dependence of the group velocity calculated from Eq. (2) with the fitting parameter $A$ of $170 \mathrm{~cm}^{-1}$ for $200 \mathrm{~nm}$ thin film and $80 \mathrm{~cm}^{-1}$ for $500 \mathrm{~nm}$ thin film. The excellent agreement exists between the calculated values of the scaled $v_{g}(\mathrm{E})$ based on Eq. (2) and the inverse of the P-emission decay time. On the basis of Eq. (2), the reflection coefficient of $R$ is calculated to be $\sim 0.99$ in both film thickness samples. This result means that the photon-like polaritons are strongly confined in the $\mathrm{ZnO}$ thin film. However, this large reflection coefficient is a curious value because the low energy side below the lowest exciton-absorption band is considered to be the transparency region for photons. Now, we focus on the group velocity of the polaritons. In the $\mathrm{ZnO}$ bulk crystal, the group velocity of exciton-photon polariton at $3.285 \mathrm{eV}$ is calculated $6.4 \times 10^{9} \mathrm{~cm} / \mathrm{s}$. On the other hand, the group velocity of that in 200 and $500 \mathrm{~nm}$ thin films is estimated $2.9 \times 10^{7} \mathrm{~cm} / \mathrm{s}$ and $3.6 \times 10^{7} \mathrm{~cm} / \mathrm{s}$, respectively, from Eq. (2) by using the observed decay time $\left(\tau_{d}\right)$ and the reported value of the reflection coefficient $(R)$ of 0.3 [5] and the sample thickness $(L)$. This result demonstrates that the group velocity of the photon-like polariton generated by the exciton-exciton scattering in the $\mathrm{ZnO}$ thin film is two orders of magnitude slower than that calculated from the exciton-polariton dispersion in $\mathrm{ZnO}$ bulk crystals.

\section{Acknowledgment}

This work was supported by JSPS KAKENHI (Grants No. 24560011).

\section{References}

1. S Chen, W Chen, and I Buyanova, Phys. Rev. B 83, 245212 (2011)

2. C. Klingshirn, and H. Haug, Phys. Rep. 70, 315 (1981)

3. J Aaviksoo, J. Lum. 48/49, 57 (1991)

4. S. Wakaiki, H. Ichida, K. Mizoguchi, D. Kim, Y. Kanematsu, and M. Nakayama, phys. stat. sol. (c), 8, $116(2011)$

5. M. Cobet, C. Cobet, M.R. Wagner, N. Esser, C. Thomsen, and A. Hoffmann, Appl. Phys. Lett. 96, 031904 (2010) 